\section{PNEUMOTHORAX AND AIR LEAK SYNDROMES IN THE NEWBORN, A COHORT STUDY}

\author{
H. Ali, S. Esampalli, L. Aldridge, M. Lal \\ Neonatal Medicine, James Cook University \\ Hospital, Middlesbrough, UK
}

Background: Pneumothorax is a common complication in the premature newborn with respiratory distress syndrome. Its incidence may have declined following the widespread use of antenatal steroids and surfactant. However clinicians are concerned that use of CPAP may be associated with increases risk of Pneumothorax.

Aim: To describe the demographic profile of a cohort of premature infants with pneumothorax.

Method: Regional tertiary neonatal centre in the north east of England. All newborns with pneumothorax or other air leak syndromes over 10 year period were included. Variables recorded; gestation, antenatal steroids, surfactant use and non-invasive respiratory support (CPAP).

Results: 3804 admissions, 107 infants were diagnosed with pneumothorax or other air leak syndrome (overall incidence of $2.8 \%$ ). This included 72 premature infants of whom 37 were under 28 weeks gestation. The incidence of pneumothorax and pulmonary interstitial emphysema (PIE) was $2.3 \%$ and $0.47 \%$ respectively. There was statistically significant association between the severity of pneumothorax and degree of prematurity, use of surfactant and CPAP. Severe pneumothorax requiring drainage was more in extremely premature infants who didn't received optimal dose of surfactant. No statistically significant effect was noted with variables such as lack of antenatal steroids, gender, mode of delivery, use of muscle relaxants and squad retrievals.

Conclusion: Incidence of pneumothorax and other air leak syndromes was lower compared with other cohort studies. Risk of pneumothorax was increased with use of CPAP and suboptimal surfactant dose. Effect of CPAP in extreme premature newborns without antenatal steroids and postnatal surfactant exposure require further evaluation.

\section{PULMONARY FUNCTION AT EARLY SCHOOL AGE FOLLOWING INTRAUTERINE GROWTH RESTRICTION WITH ABNORMAL FETAL BLOOD FLOW AND VERY PRETERM BIRTH}

\author{
E.K. Morsing ${ }^{1}$, P. Gustafsson ${ }^{2}$, K. Marsal ${ }^{3}$, \\ J. Brodszki ${ }^{3}$
}

${ }^{1}$ Department of Pediatrics, University Hospital in Lund, Lund, ${ }^{2}$ Department of Pediatrics, Central Hospital, Skövde, ${ }^{3}$ Department of Obstetrics and Gynecology, University Hospital of Lund, Lund, Sweden

Background: There is evidence of impaired lung development during childhood following preterm birth. Infants born very preterm due to intrauterine growth restriction (IUGR) and absent or reversed end-diastolic blood flow (ARED) have an increased risk for chronic lung disease (CLD).

Objective: To evaluate pulmonary function at early school age in children born at very early gestation due to IUGR and ARED.

Methods: Data on self-reported respiratory health and lung function obtained by spirometry were evaluated in 19 children (PT-IUGR) with IUGRARED with a mean (SD) birthweight of 681(125) $\mathrm{g}$ born at median (range) 27(24-29) gestational weeks. These were compared with 19 children (PT-AGA) matched for gestational age at birth, gender and time of delivery with a BW appropriate for gestational age at birth (AGA), 1082(298) g and with 19 children (T-AGA) born at term with BW AGA 3647 (465) g matched for gender and time of delivery. Spirometry was recorded at age 7.3 to 10.7 yrs (median 9.0).

Results: The PT-IUGR group had lower mean (SD) values of \% predicted Forced Expiratory Volume, $1 \mathrm{sec}\left(\mathrm{FEV}_{1}\right)$ and Forced Expiratory Flow, 25-75\% $\left(\mathrm{FEF}_{25-75}\right)$ compared to the T-AGA group. $\mathrm{FEV}_{1}: 85.4$ (13.0) and 96.6 (16.1) $p=0.046$ and $\mathrm{FEF}_{25-75}: 67.7$ (18.0) and 92.3 (28.5), $p=0.006$ respectively. The PT-AGA group did not differ from the T-AGA group. Self-reported rate of asthma did not differ between groups.

Conclusion: At early school age, pulmonary function is particularly impaired in children born preterm with IUGR-ARED suggesting additional detrimental effect on lung development. 